\title{
On Factorizations of Upper Triangular Nonnegative Matrices of Order Three
}

\author{
Yi-Zhi Chen \\ Department of Mathematics, Huizhou University, Huizhou, Guangdong 516007, China \\ Correspondence should be addressed to Yi-Zhi Chen; yizhichen1980@126.com
}

Received 2 February 2015; Revised 18 March 2015; Accepted 18 March 2015

Academic Editor: Ivan Area

Copyright (C) 2015 Yi-Zhi Chen. This is an open access article distributed under the Creative Commons Attribution License, which permits unrestricted use, distribution, and reproduction in any medium, provided the original work is properly cited.

Let $T_{3}\left(\mathbb{N}_{0}\right)$ denote the semigroup of $3 \times 3$ upper triangular matrices with nonnegative integral-valued entries. In this paper, we investigate factorizations of upper triangular nonnegative matrices of order three. Firstly, we characterize the atoms of the subsemigroup $S$ of the matrices in $T_{3}\left(\mathbb{N}_{0}\right)$ with nonzero determinant and give some formulas. As a consequence, problems $4 \mathrm{a}$ and $4 \mathrm{c}$ presented by Baeth et al. (2011) are each half-answered for the case $n=3$. And then, we consider some factorization cases of matrix $A$ in $S$ with $\rho(A)=1$ and give formulas for the minimum factorization length of some special matrices in $S$.

\section{Introduction and Preliminaries}

Upper triangular matrices are an important class of matrices, which lead to a broader study of all integral-valued matrices. There are many papers in the literature considering these matrices and similar topics.

Factoring such matrices plays a vital role in the study of upper triangular matrices (see [1]). The problem of factoring matrices was studied by Cohn [2] as early as 1963. Later, Jacobson and Wisner $[3,4]$ and Ch'uan and Chuan $[5,6]$ investigated these factorization problems in the context of semigroups of matrices. Motivated by these results, Baeth et al. [7] applied the concepts of contemporary factorization theory to semigroups of integral-valued matrices and calculated certain important invariants to give a sense of how unique or nonunique factorization is in each of these semigroups. In [7], Baeth et al. presented six open problems.

In this paper, we will investigate factorizations of upper triangular nonnegative matrices of order three. Also, we will consider open problem 4 presented in [7].

Throughout this paper, $\mathbb{N}$ will denote the set of all positive integers and $\mathbb{N}_{0}=\mathbb{N} \cup\{0\}$. Also, $T_{3}\left(\mathbb{N}_{0}\right)$ will denote the semigroup of $3 \times 3$ upper triangular matrices with nonnegative integral-valued entries.

In the following, analogous with [7] or [8], some concepts and preliminaries are recalled.
A semigroup is a pairing $(S, \cdot)$ where $S$ is a set and $\cdot$ is an associative binary operation on $S$. When the binary operation is clear from context and $A, B \in S$, we will simply write $A B$ instead of $A \cdot B$. If $S$ contains an element $I$ such that $A I=I A=$ $A$ for all $A \in S$, then $I$ is the identity of $S$.

Let $S$ be a semigroup with identity $I$. An element $A \in S$ is a unit of $S$ if there exists an element $B \in S$ such that $A B=B A=$ I. A nonunit $A \in S$ is called an atom of $S$ if whenever $A=B C$ for some elements $B, C \in S$, either $B$ or $C$ is a unit of $S$. The semigroup $S$ is said to be atomic provided each nonunit element in $S$ can be written as a product of atoms of $S$.

Let $S$ denote an atomic semigroup and let $A$ be a nonunit element of $S$. The set

$\mathscr{L}(A)=\left\{t: A=A_{1} A_{2} \cdots A_{t}\right.$ with each $A_{i}$ an atom of $\left.S\right\}$

is called the set of lengths of $A$.

We denote by $L(A)=\sup \mathscr{L}(A)$ the longest (if finite) factorization length of $A$ and $l(A)=\min \mathscr{L}(A)$ the minimum factorization length of $A$. The elasticity of $A$, denoted by

$$
\rho(A)=\frac{L(A)}{l(A)},
$$


gives a coarse measure of how far away $A$ is from having unique factorization. It is not hard to see that if $A$ has a unique factorization $A=A_{1} A_{2} \cdots A_{t}$, whence $\mathscr{L}(A)=\{t\}$ and so

$$
l(A)=L(A)=t, \quad \rho(A)=\frac{t}{t}=1 .
$$

The elasticity of the semigroup $S$, denoted by $\rho(S)$, is given by

$$
\rho(S)=\sup \{\rho(A): A \in S\} .
$$

If $\mathscr{L}(A)=\left\{t_{1}, t_{2}, \ldots\right\}$ with $t_{i}<t_{i+1}$ for each $i$, then the delta set of $A$ is given by

$$
\Delta(A)=\left\{t_{i+1}-t_{i}: t_{i}, t_{i+1} \in \mathscr{L}(A)\right\}
$$

and $\Delta(S)=\cup_{A \in S} \Delta(A)$.

This paper will be divided into two sections. In Section 2 , we will consider the semigroup $S$ of upper triangular $3 \times 3$ matrices with nonnegative entries and nonzero determinant. Firstly, we characterize the atoms of the subsemigroup $S$ of the matrices in $T_{3}\left(\mathbb{N}_{0}\right)$ with nonzero determinant and give some formulas. As a result, problems $4 \mathrm{a}$ and $4 \mathrm{c}$ presented by Baeth et al. in [7] are each half-answered for the case $n=3$. And then, we consider some factorization cases of matrix $A$ in $S$ with $\rho(A)=1$ and give formulas for the minimum factorization length of some special matrices in $S$.

\section{Upper Triangular Nonnegative Matrices of Order Three}

In this section we consider the semigroup $S$ of upper triangular $3 \times 3$ matrices with nonnegative entries and nonzero determinant. In this case, $I_{3}$ is the only unit of $S$.

For each pair $i, j \in\{1,2,3\}$, let $E_{i, j}$ denote the matrix whose only nonzero entry is $e_{i j}=1$.

The following theorem gives some characterizations about the atoms of $S$.

Theorem 1. Let $S$ denote the subsemigroup of the matrices in $T_{3}\left(\mathbb{N}_{0}\right)$ with nonzero determinant. The set of atoms of $S$ consists of the matrices

$$
\begin{gathered}
X_{12}=\left(\begin{array}{lll}
1 & 1 & 0 \\
0 & 1 & 0 \\
0 & 0 & 1
\end{array}\right), \quad X_{13}=\left(\begin{array}{lll}
1 & 0 & 1 \\
0 & 1 & 0 \\
0 & 0 & 1
\end{array}\right) \\
\text { or } X_{23}=\left(\begin{array}{lll}
1 & 0 & 0 \\
0 & 1 & 1 \\
0 & 0 & 1
\end{array}\right)
\end{gathered}
$$

and, for each prime $p$, the matrices

$$
\begin{gathered}
Y_{11}=\left(\begin{array}{lll}
p & 0 & 0 \\
0 & 1 & 0 \\
0 & 0 & 1
\end{array}\right), \quad Y_{22}=\left(\begin{array}{lll}
1 & 0 & 0 \\
0 & p & 0 \\
0 & 0 & 1
\end{array}\right) \\
\text { or } Y_{33}=\left(\begin{array}{lll}
1 & 0 & 0 \\
0 & 1 & 0 \\
0 & 0 & p
\end{array}\right) .
\end{gathered}
$$

Proof. Suppose that $X_{i j}=X_{1} X_{2}$ for some $X_{1}, X_{2} \in S$. Since $\operatorname{det}\left(X_{i j}\right)=1, \operatorname{det}\left(X_{1}\right)=\operatorname{det}\left(X_{2}\right)=1$ and we can write

$$
X_{i j}=X_{1} X_{2}=\left(\begin{array}{ccc}
1 & b_{12} & b_{13} \\
0 & 1 & b_{23} \\
0 & 0 & 1
\end{array}\right)\left(\begin{array}{ccc}
1 & c_{12} & c_{13} \\
0 & 1 & c_{23} \\
0 & 0 & 1
\end{array}\right),
$$

where $\sum_{k=1}^{3} b_{i k} c_{k j}=1$ and $\sum_{k=1}^{3} b_{h k} c_{k l}=0$ if $h \neq i$ and $l \neq j$. As a result, either $X_{1}$ or $X_{2}$ is the identity and hence $X_{i j}$ is an atom.

Note that the proofs that $Y_{i i}(1 \leq i \leq 3)$ are atoms are similar; we only prove that $Y_{22}$ is an atom in the following. Suppose now that $p$ is prime and $Y_{22}=Y_{1} Y_{2}, Y_{1}, Y_{2} \in S$. Since $p$ is prime, either

$$
Y_{1} Y_{2}=\left(\begin{array}{ccc}
1 & b_{12} & b_{13} \\
0 & p & b_{23} \\
0 & 0 & 1
\end{array}\right)\left(\begin{array}{ccc}
1 & c_{12} & c_{13} \\
0 & 1 & c_{23} \\
0 & 0 & 1
\end{array}\right)
$$

or

$$
Y_{1} Y_{2}=\left(\begin{array}{ccc}
1 & b_{12} & b_{13} \\
0 & 1 & b_{23} \\
0 & 0 & 1
\end{array}\right)\left(\begin{array}{ccc}
1 & c_{12} & c_{13} \\
0 & p & c_{23} \\
0 & 0 & 1
\end{array}\right)
$$

In either case, $b_{i j}=c_{i j}=0$ for $1 \leq i<j \leq 3$. Consequently, either $Y_{1}$ or $Y_{2}$ is the identity and hence $Y_{i i}$ is an atom of $S$.

Finally, we will show that these are the only atoms of $S$.

For any

$$
A=\left(\begin{array}{ccc}
a_{11} & a_{12} & a_{13} \\
0 & a_{22} & a_{23} \\
0 & 0 & a_{33}
\end{array}\right) \in S
$$

we can write

$$
\begin{aligned}
A= & {\left[I_{3}+\left(a_{33}-1\right) E_{3,3}\right]\left[I_{3}+a_{23} E_{2,3}\right]\left[I_{3}+a_{13} E_{1,3}\right] } \\
& \cdot\left[I_{3}+\left(a_{22}-1\right) E_{2,2}\right]\left[I_{3}+a_{12} E_{1,2}\right]\left[I_{3}+\left(a_{11}-1\right) E_{1,1}\right] \\
= & \left(\begin{array}{lll}
1 & 0 & 0 \\
0 & 1 & 0 \\
0 & 0 & a_{33}
\end{array}\right)\left(\begin{array}{ccc}
1 & 0 & 0 \\
0 & 1 & a_{23} \\
0 & 0 & 1
\end{array}\right)\left(\begin{array}{ccc}
1 & 0 & a_{13} \\
0 & 1 & 0 \\
0 & 0 & 1
\end{array}\right) \\
& \cdot\left(\begin{array}{lll}
1 & 0 & 0 \\
0 & a_{22} & 0 \\
0 & 0 & 1
\end{array}\right)\left(\begin{array}{ccc}
1 & a_{12} & 0 \\
0 & 1 & 0 \\
0 & 0 & 1
\end{array}\right)\left(\begin{array}{ccc}
a_{11} & 0 & 0 \\
0 & 1 & 0 \\
0 & 0 & 1
\end{array}\right) .
\end{aligned}
$$

Thus, the set of atoms of $S$ consists of the matrices $A=I_{3}+E_{i, j}$ for each pair $i$ and $j$ with $1<i<j<3$ or $A=I_{3}+(p-1) E_{i, i}$ for some prime $p$ and $1 \leq i \leq 3$.

Recall that a unitriangular matrix is a matrix in $T_{3}\left(\mathbb{N}_{0}\right)$ whose diagonal elements are all 1s. Denote $\Sigma(A)=$ $\Sigma_{1 \leq i<j \leq 3} a_{i j}$. From the proof of Theorem 1, we can immediately obtain the following corollary. 
Corollary 2. Let $S$ denote the unitriangular matrices in $T_{3}\left(\mathbb{N}_{0}\right)$ and $A \in S$. Then $A$ is an atom if and only if $\Sigma(A)=1$.

Hereafter, for any given $A \in T_{3}\left(\mathbb{N}_{0}\right)$ with nonzero determinant, we let $r(A)$ denote the number of (not necessarily distinct) prime factors of $\operatorname{det}(A)$.

Proposition 3. Let $S$ denote the subsemigroup of the matrices in $T_{3}\left(\mathbb{N}_{0}\right)$ with nonzero determinant. If $A$ can be factored as $A=A_{1} A_{2} \cdots A_{t}$ with each $A_{i}$ being an atom of $S$, then $t=$ $r(A)+k$, where

$k=\left|\left\{i: A_{i} \in\left\{\left(\begin{array}{lll}1 & 1 & 0 \\ 0 & 1 & 0 \\ 0 & 0 & 1\end{array}\right),\left(\begin{array}{lll}1 & 0 & 1 \\ 0 & 1 & 0 \\ 0 & 0 & 1\end{array}\right),\left(\begin{array}{lll}1 & 0 & 0 \\ 0 & 1 & 1 \\ 0 & 0 & 1\end{array}\right)\right\}\right\}\right|$

Proof. For each $i, A_{i}$ is an atom and thus $\operatorname{det}\left(A_{i}\right)$ is either 1 or prime. Since

$$
\operatorname{det}(A)=\operatorname{det}\left(A_{1}\right) \operatorname{det}\left(A_{2}\right) \cdots \operatorname{det}\left(A_{t}\right) \text {, }
$$

we have

$$
\mid\left\{i: \operatorname{det}\left(A_{i}\right) \text { is prime }\right\} \mid=r(A) .
$$

If $k=\left|\left\{i: \operatorname{det}\left(A_{i}\right)=1\right\}\right|$, then the length of this factorization of $A$ is

$$
\begin{aligned}
t & =\mid\left\{i: \operatorname{det}\left(A_{i}\right) \text { is prime }\right\}|+|\left\{i: \operatorname{det}\left(A_{i}\right)=1\right\} \mid \\
& =r(A)+k .
\end{aligned}
$$

Lemma 4 (see [7, Theorem 4.4]). Let $S$ denote the subsemigroup of $T_{n}\left(\mathbb{N}_{0}\right)$ of unitriangular matrices and let $A \in S$. Then $L(A)=\Sigma(A)$.

Theorem 5. Let $S$ denote the subsemigroup of the matrices in $T_{3}\left(\mathbb{N}_{0}\right)$ with nonzero determinant and

$$
A=\left(\begin{array}{ccc}
a_{11} & a_{12} & a_{13} \\
0 & a_{22} & a_{23} \\
0 & 0 & a_{33}
\end{array}\right) \in S
$$

Then the following statements hold:

(1) $L(A)=r(A)+a_{12}+a_{13}+a_{23}$;

(2) if $a_{12}=a_{13}=a_{23}=0$, then $l(A)=r(A)=L(A)$ and $\rho(A)=1$;

(3) if $a_{12} \mid a_{11} a_{22}\left(a_{12}>0\right), a_{13}=a_{23}=0$, and then

$$
l(A)=r(A)+1, \quad \rho(A)=\frac{r(A)+a_{12}}{r(A)+1} ;
$$

(4) if $a_{13} \mid a_{11} a_{33}\left(a_{13}>0\right), a_{12}=a_{23}=0$, and then

$$
l(A)=r(A)+1, \quad \rho(A)=\frac{r(A)+a_{13}}{r(A)+1} ;
$$

(5) if $a_{23} \mid a_{22} a_{33}\left(a_{23}>0\right), a_{12}=a_{13}=0$, and then

$$
l(A)=r(A)+1, \quad \rho(A)=\frac{r(A)+a_{23}}{r(A)+1} .
$$

Proof. (1) Suppose that $A=A_{1} A_{2} \cdots A_{t}$ with each $A_{i}$ being an atom of $S$. By Proposition $3, t=r(A)+k$ where

$$
k=\left|\left\{i: A_{i} \in\left\{I_{3}+E_{12}, I_{3}+E_{13}, I_{3}+E_{23}\right\}\right\}\right| .
$$

Note that the numbers of factors $I_{3}+E_{12}\left(I_{3}+E_{13}, I_{3}+E_{23}\right.$, resp.) of $A$ are not more than $a_{12}\left(a_{13}, a_{23}\right.$, resp.). Then we have

$$
k \leq a_{12}+a_{13}+a_{23}=\Sigma(A)
$$

and thus

$$
L(A) \leq r(A)+\sum(A) .
$$

On the other hand, from the proof of Theorem 1, we know that

$$
\begin{aligned}
A= & A_{1} A_{2} A_{3} A_{4} A_{5} A_{6} \\
= & {\left[I_{3}+\left(a_{33}-1\right) E_{3,3}\right]\left[I_{3}+a_{23} E_{2,3}\right] } \\
& \cdot\left[I_{3}+a_{13} E_{1,3}\right]\left[I_{3}+\left(a_{22}-1\right) E_{2,2}\right]\left[I_{3}+a_{12} E_{1,2}\right] \\
& \cdot\left[I_{3}+\left(a_{11}-1\right) E_{1,1}\right],
\end{aligned}
$$

and then,

$$
\begin{aligned}
L(A) \geq & L\left(A_{1}\right)+L\left(A_{2}\right)+L\left(A_{3}\right)+L\left(A_{4}\right) \\
& +L\left(A_{5}\right)+L\left(A_{6}\right) \\
= & r\left(A_{1}\right)+r\left(A_{4}\right)+r\left(A_{6}\right)+L\left(A_{2}\right)+L\left(A_{3}\right)+L\left(A_{5}\right) \\
= & r(A)+\sum_{1 \leq i<j \leq n} a_{i j}=r(A)+\sum(A) .
\end{aligned}
$$

Thus, combining (23) and (25), we have $L(A)=r(A)+\Sigma(A)$.

(2) Suppose that $A$ is a diagonal matrix; that is, $a_{i j}=0$ for all $i, j \in\{1,2,3\}$ with $i<j$, and write $t=r(A)+k$ as in Proposition 3. If $k \geq 1$, then $A$ contains at least one factor of $X_{12}=I_{3}+E_{12}, X_{13}=I_{3}+E_{13}$, or $X_{23}=I_{3}+E_{23}$, and then there is at least one superdiagonal entry of $A$ that is not 0 . This contradicts with the fact that $A$ is a diagonal matrix. Thus, $t=r(A)=l(A)=L(A)$ and, in this case,

$$
\rho(A)=\frac{L(A)}{l(A)}=1
$$

(3) Suppose that $a_{12} \mid a_{11} a_{22}\left(a_{12}>0\right), a_{13}=a_{23}=0$. Denote $l(A)=r(A)+k$ as in Proposition 3. Notice that $r(A)$ is the number of (not necessarily distinct) prime factors of $\operatorname{det}(A)$ and $a_{12}>0$. Then $k \geq 1$, and so $l(A) \geq r(A)+1$. Since $a_{12}>0, a_{12} \mid a_{11} a_{22}, a_{12}$ can be factored as a product of one factor of $a_{11}$ and one factor of $a_{22}$; say $a_{12}=a_{11}^{\prime} a_{22}^{\prime}$ where 
$a_{11}=m_{11} a_{11}^{\prime}$ and $a_{22}=n_{22} a_{22}^{\prime}$ for some positive integers $m_{11}$ and $n_{22}$. Factor $A$ as

$$
\begin{aligned}
A= & A_{1} A_{2} \cdots A_{8} \\
= & \left(\begin{array}{ccc}
a_{33}^{\prime} & 0 & 0 \\
0 & 1 & 0 \\
0 & 0 & 1
\end{array}\right)\left(\begin{array}{ccc}
1 & 0 & 0 \\
0 & n_{22} & 0 \\
0 & 0 & 1
\end{array}\right)\left(\begin{array}{lll}
1 & 1 & 0 \\
0 & 1 & 0 \\
0 & 0 & 1
\end{array}\right)\left(\begin{array}{lll}
1 & 0 & 0 \\
0 & 1 & 0 \\
0 & 0 & a_{33}
\end{array}\right) \\
& \cdot\left(\begin{array}{lll}
1 & 0 & 0 \\
0 & a_{22} & 0 \\
0 & 0 & 1
\end{array}\right)\left(\begin{array}{ccc}
a_{11} & 0 & 0 \\
0 & 1 & 0 \\
0 & 0 & 1
\end{array}\right)\left(\begin{array}{ccc}
m_{11} & 0 & 0 \\
0 & 1 & 0 \\
0 & 0 & 1
\end{array}\right) \\
& \left(\begin{array}{lll}
1 & 0 & 0 \\
0 & a_{22}^{\prime} & 0 \\
0 & 0 & 1
\end{array}\right) .
\end{aligned}
$$

By the above factorization of $A$ and (2), it is not hard to see that

$$
l(A) \leq l\left(A_{1}\right)+l\left(A_{2}\right)+\cdots+l\left(A_{8}\right)=r(A)+1 .
$$

Thus, $l(A)=r(A)+1$.

In this case we immediately get

$$
\rho(A)=\frac{r(A)+\Sigma(A)}{r(A)+1}=\frac{r(A)+a_{12}}{r(A)+1} .
$$

Hence, (3) holds.

Similarly, we can show that (4) and (5) hold.

Remark 6. Given $A \in S$, Theorem 5 provides a formula for $L(A)$. Consequently, open problems $4 \mathrm{a}$ and $4 \mathrm{c}$ in [7] are each half-answered for the case $n=3$.

Theorem 7. Let $S$ denote the subsemigroup of the matrices in $T_{3}\left(\mathbb{N}_{0}\right)$ with nonzero determinant and

$$
A=\left(\begin{array}{ccc}
a_{11} & a_{12} & a_{13} \\
0 & a_{22} & a_{23} \\
0 & 0 & a_{33}
\end{array}\right) \in S \text {. }
$$

If A satisfies one of the following conditions,

(1) $a_{12}=a_{13}=a_{23}=0$;

(2) $a_{11}=a_{22}=a_{33}=1, a_{12}=0$;

(3) $a_{11}=a_{22}=a_{33}=1, a_{13}=0$;

(4) $a_{11}=a_{22}=a_{33}=1, a_{23}=0$;

(5) $a_{12}=a_{23}=1, a_{13}=0$;

(6) $a_{12}=a_{23}=1, a_{23}=0$;

(7) $a_{13}=a_{23}=1, a_{12}=0$,
Proof. (1) If $a_{12}=a_{13}=a_{23}=0$, by Theorem $5, l(A)=r(A)=$ $L(A)$. In this case, $\rho(A)=1$.

(2) Suppose that $a_{11}=a_{22}=a_{33}=1$ and $a_{12}=0$. If $a_{13}=$ $a_{23}=0$, by (1), the conclusion holds. Now, assume that $a_{13}=$ $a_{23} \neq 0$; the cases of that $a_{13}=0, a_{23} \neq 0$ or $a_{13} \neq 0, a_{23}=$ 0 can be proved similarly. In this case, $X_{12}$ is not an atomic factor of $A$ and $X_{13}$ and $X_{23}$ are atomic factors of $A$. Note that $X_{13}$ can commute with $X_{23}$; then $A$ only has the following factorization:

$$
A=\left(\begin{array}{lll}
1 & 0 & 1 \\
0 & 1 & 0 \\
0 & 0 & 1
\end{array}\right)^{a_{13}} \cdot\left(\begin{array}{lll}
1 & 0 & 0 \\
0 & 1 & 1 \\
0 & 0 & 1
\end{array}\right)^{a_{23}}
$$

with $\rho(A)=1$ and the minimum factorization length $l(A)=$ $a_{13}+a_{23}$.

Analogous with the proof of (2), we can show that (4) holds.

(3) Suppose that $a_{11}=a_{22}=a_{33}=1$ and $a_{13}=0$. If $a_{12}=$ $a_{23}=0$, by (1), the conclusion holds. Now, assume that $a_{12}=$ $a_{23} \neq 0$; the cases of that $a_{12}=0, a_{23} \neq 0$ or $a_{12} \neq 0, a_{23}=$ 0 can be proved similarly. In this case, $X_{13}$ is not an atomic factor of $A$ and $X_{12}$ and $X_{23}$ are atomic factors of $A$. Note that $X_{12} X_{23}=X_{23} X_{12} X_{13}$; the factorizations of $A$ must satisfy that all the $X_{23}$ matrices are before $X_{12}$ matrices, and then $A$ only has the following factorization:

$$
A=\left(\begin{array}{lll}
1 & 0 & 0 \\
0 & 1 & 1 \\
0 & 0 & 1
\end{array}\right)^{a_{23}} \cdot\left(\begin{array}{lll}
1 & 1 & 0 \\
0 & 1 & 0 \\
0 & 0 & 1
\end{array}\right)^{a_{12}}
$$

with $\rho(A)=1$ and the minimum factorization length $l(A)=$ $a_{12}+a_{23}$.

(5) Suppose that $a_{12}=a_{23}=1$ and $a_{13}=0$. In this case, $X_{13}$ is not an atomic factor of $A$, and $X_{12}$ and $X_{23}$ are atomic factors of $A$. Assume that $A$ can be factored as $A=$ $A_{1} A_{2} \cdots A_{t}$ with each $A_{i}$ being an atom; then $t=r(A)+k$, where

$$
k=\left|\left\{i: A_{i} \in\left\{X_{12}, X_{23}\right\}\right\}\right| .
$$

By Theorem 5 (1), $k \leq a_{12}+a_{23}+a_{13}=2$. On the other hand, since $a_{12}=a_{23}=1, X_{12}, X_{23}$ are atomic factors of $A$, and we have $k \geq 2$. Thus, $k=2$. In this case, $A$ can factorize as follows:

$$
\begin{aligned}
A= & {\left[I_{3}+\left(a_{33}-1\right) E_{3,3}\right]\left[I_{3}+E_{2,3}\right]\left[I_{3}+\left(a_{22}-1\right) E_{2,2}\right] } \\
& \cdot\left[I_{3}+E_{1,2}\right]\left[I_{3}+\left(a_{11}-1\right) E_{1,1}\right] \\
= & \left(\begin{array}{lll}
1 & 0 & 0 \\
0 & 1 & 0 \\
0 & 0 & a_{33}
\end{array}\right)\left(\begin{array}{lll}
1 & 0 & 0 \\
0 & 1 & 1 \\
0 & 0 & 1
\end{array}\right)\left(\begin{array}{ccc}
1 & 0 & 0 \\
0 & a_{22} & 0 \\
0 & 0 & 1
\end{array}\right) \\
& \cdot\left(\begin{array}{lll}
1 & 1 & 0 \\
0 & 1 & 0 \\
0 & 0 & 1
\end{array}\right)\left(\begin{array}{ccc}
a_{11} & 0 & 0 \\
0 & 1 & 0 \\
0 & 0 & 1
\end{array}\right) .
\end{aligned}
$$

then $\rho(A)=1$. 
So if we give the atomic factorizations of $\left[I_{3}+\left(a_{33}-1\right) E_{3,3}\right]$, $\left[I_{3}+\left(a_{22}-1\right) E_{2,2}\right]$, and $\left[I_{3}+\left(a_{11}-1\right) E_{1,1}\right]$, then we will obtain the atomic factorization of $A$ with $\rho(A)=1$ and the minimum factorization length $l(A)=r(A)+2$.

Analogous with the proof of (5), we can show that (6) and (7) hold.

Example 8. Consider

$$
A=\left(\begin{array}{lll}
1 & 0 & 2 \\
0 & 1 & 3 \\
0 & 0 & 1
\end{array}\right) \in T_{3}\left(\mathbb{N}_{0}\right)
$$

Then by Theorem 7 (2), we can obtain the atomic factorization of $A$ with $\rho(A)=1$ and the minimum factorization length $l(A)=2+3=5$ as follows:

$$
A=\left(\begin{array}{lll}
1 & 0 & 1 \\
0 & 1 & 0 \\
0 & 0 & 1
\end{array}\right)^{2} \cdot\left(\begin{array}{lll}
1 & 0 & 0 \\
0 & 1 & 1 \\
0 & 0 & 1
\end{array}\right)^{3}
$$

Example 9. Consider

$$
A=\left(\begin{array}{lll}
6 & 1 & 0 \\
0 & 4 & 1 \\
0 & 0 & 8
\end{array}\right) \in T_{3}\left(\mathbb{N}_{0}\right)
$$

Then by Theorem 7 (5), we can obtain the atomic factorization of $A$ with $\rho(A)=1$ and the minimum factorization length $l(A)=r(A)+2=9$ as follows:

$$
\begin{aligned}
A= & \left(\begin{array}{lll}
1 & 0 & 0 \\
0 & 1 & 0 \\
0 & 0 & 2
\end{array}\right)^{3}\left(\begin{array}{lll}
1 & 0 & 0 \\
0 & 1 & 1 \\
0 & 0 & 1
\end{array}\right)\left(\begin{array}{lll}
1 & 0 & 0 \\
0 & 2 & 0 \\
0 & 0 & 1
\end{array}\right)^{2} \\
& \cdot\left(\begin{array}{lll}
1 & 0 & 0 \\
0 & 1 & 1 \\
0 & 0 & 1
\end{array}\right)\left(\begin{array}{lll}
2 & 0 & 0 \\
0 & 1 & 0 \\
0 & 0 & 1
\end{array}\right)\left(\begin{array}{lll}
3 & 0 & 0 \\
0 & 1 & 0 \\
0 & 0 & 1
\end{array}\right) .
\end{aligned}
$$

Theorem 10. Let $S$ denote the unitriangular matrices in $T_{3}\left(\mathbb{N}_{0}\right)$ and

$$
A=\left(\begin{array}{ccc}
1 & a_{12} & a_{13} \\
0 & 1 & a_{23} \\
0 & 0 & 1
\end{array}\right) \in S, \quad a_{12}, a_{13}, a_{23}>0
$$

Then we have

$$
l(A)= \begin{cases}a_{12}+a_{23}, & \text { if } a_{13} \leq a_{12} a_{23}, \\ a_{12}+a_{23}+a_{13}-a_{12} a_{23}, & \text { if } a_{13} \geq a_{12} a_{23} .\end{cases}
$$

Proof. It is a routine way to check that $X_{12} X_{13}=X_{13} X_{12}$, $X_{23} X_{13}=X_{13} X_{23}$, and $X_{12} X_{23}=X_{23} X_{13} X_{12}$. Hence the maximum length is achieved by putting all the $X_{23}$ matrices before the $X_{12}$ matrices. In this case, we have the form
$X_{23}^{a_{23}} X_{12}^{a_{12}} X_{13}^{a_{13}}$. The minimum length is achieved by having as few as possible $X_{13}$ matrices, that is, by putting $X_{23}$ matrices after $X_{12}$ matrices. If $a_{13} \leq a_{12} a_{23}$, we can run out of $X_{13}$ matrices, leaving a form $X_{12}^{a_{12}^{\prime}} X_{23}^{a_{23}^{\prime}} X_{12} X_{23}^{a_{23}^{\prime \prime}} X_{12}^{a_{12}^{\prime \prime}}$, where these powers satisfy $a_{12}^{\prime}+1+a_{12}^{\prime \prime}=a_{12}, a_{23}^{\prime}+a_{23}^{\prime \prime}=a_{23}$, and $a_{12}^{\prime} a_{23}+$ $a_{23}^{\prime \prime}=a_{13}$. And, in this case, we have $l(A)=a_{12}+a_{23}$. If $a_{13} \geq$ $a_{12} a_{23}$, then we can put all the $X_{23}$ matrices after all of the $X_{12}$ matrices, getting the form $X_{12}^{a_{12}} X_{23}^{a_{23}} X_{13}^{a_{13}-a_{12} a_{23}}$. Thus, in this case, we have $l(A)=a_{12}+a_{23}+a_{13}-a_{12} a_{23}$.

Example 11. Consider

$$
A=\left(\begin{array}{lll}
1 & 2 & 2 \\
0 & 1 & 3 \\
0 & 0 & 1
\end{array}\right) \in T_{3}\left(\mathbb{N}_{0}\right)
$$

Then by Theorem 10, we can obtain a factorization of $A$ with the minimum factorization length $l(A)=a_{12}+a_{23}=5$ as follows:

$$
A=\left(\begin{array}{lll}
1 & 0 & 0 \\
0 & 1 & 1 \\
0 & 0 & 1
\end{array}\right)^{2}\left(\begin{array}{lll}
1 & 1 & 0 \\
0 & 1 & 0 \\
0 & 0 & 1
\end{array}\right)^{2}\left(\begin{array}{lll}
1 & 1 & 0 \\
0 & 1 & 0 \\
0 & 0 & 1
\end{array}\right) .
$$

Example 12. Consider

$$
A=\left(\begin{array}{lll}
1 & 2 & 6 \\
0 & 1 & 3 \\
0 & 0 & 1
\end{array}\right) \in T_{3}\left(\mathbb{N}_{0}\right)
$$

Then by Theorem 10, we can obtain a factorization of $A$ with the minimum factorization length $l(A)=a_{12}+a_{23}=5$ as follows:

$$
A=\left(\begin{array}{lll}
1 & 1 & 0 \\
0 & 1 & 0 \\
0 & 0 & 1
\end{array}\right)^{2}\left(\begin{array}{lll}
1 & 0 & 0 \\
0 & 1 & 1 \\
0 & 0 & 1
\end{array}\right)^{3}
$$

Example 13. Consider

$$
A=\left(\begin{array}{lll}
1 & 2 & 7 \\
0 & 1 & 3 \\
0 & 0 & 1
\end{array}\right) \in T_{3}\left(\mathbb{N}_{0}\right)
$$

Then by Theorem 10, we can obtain a factorization of $A$ with the minimum factorization length $l(A)=a_{12}+a_{23}+a_{13}-$ $a_{12} a_{23}=6$ as follows:

$$
A=\left(\begin{array}{lll}
1 & 1 & 0 \\
0 & 1 & 0 \\
0 & 0 & 1
\end{array}\right)^{2}\left(\begin{array}{lll}
1 & 0 & 0 \\
0 & 1 & 1 \\
0 & 0 & 1
\end{array}\right)^{3}\left(\begin{array}{lll}
1 & 0 & 1 \\
0 & 1 & 0 \\
0 & 0 & 1
\end{array}\right)
$$

\section{Conflict of Interests}

The author declares that there is no conflict of interests regarding the publication of this paper. 


\section{Acknowledgments}

The author would like to extend their sincere gratitude to the referee for his instructive advice and useful suggestions that contributed to this paper. This research was supported by grants of the NNSF of China (nos. 11401246, 11426112), the NSF of Guangdong Province (nos. 2014A030310087, 2014A030310119), the NSF of Fujian Province (no. 2014J01019), the Outstanding Young Innovative Talent Training Project in Guangdong Universities (no. 2013LYM0086), and Science and Technology Plan Project of Huizhou City.

\section{References}

[1] V. Halava and T. Harju, “On Markov's undecidability theorem for integer matrices," Semigroup Forum, vol. 75, no. 1, pp. 173180, 2007.

[2] P. M. Cohn, "Noncommutative unique factorization domains," Transactions of the American Mathematical Society, vol. 109, pp. 313-331, 1963.

[3] B. Jacobson, "Matrix number theory: an example of nonunique factorization," The American Mathematical Monthly, vol. 72, no. 4, pp. 399-402, 1965.

[4] B. Jacobson and R. J. Wisner, "Matrix number theory I. Factorization of $2 \times 2$ unimodular matrices," Publicationes Mathematicae Debrecen, vol. 13, pp. 67-72, 1966.

[5] J. C. Ch'uan and W. F. Chuan, "Factorizations in a semigroup of integral matrices," Linear and Multilinear Algebra, vol. 18, no. 3, pp. 213-223, 1985.

[6] J. C. Ch'uan and W. F. Chuan, "Factorability of positive-integral matrices of prime determinants," Bulletin of the Institute of Mathematics. Academia Sinica, vol. 14, no. 1, pp. 11-20, 1986.

[7] N. Baeth, V. Ponomarenko, D. Adams et al., "Number theory of matrix semigroups," Linear Algebra and Its Applications, vol. 434, no. 3, pp. 694-711, 2011.

[8] D. Adams, R. Ardila, D. Hannasch et al., "Bifurcus semigroups and rings," Involve, vol. 2, no. 3, pp. 351-356, 2009. 


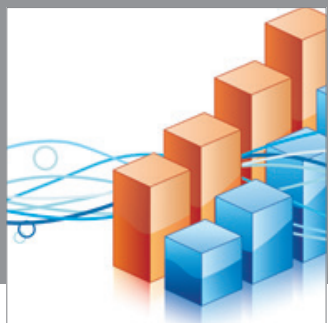

Advances in

Operations Research

mansans

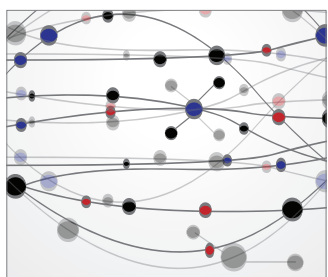

The Scientific World Journal
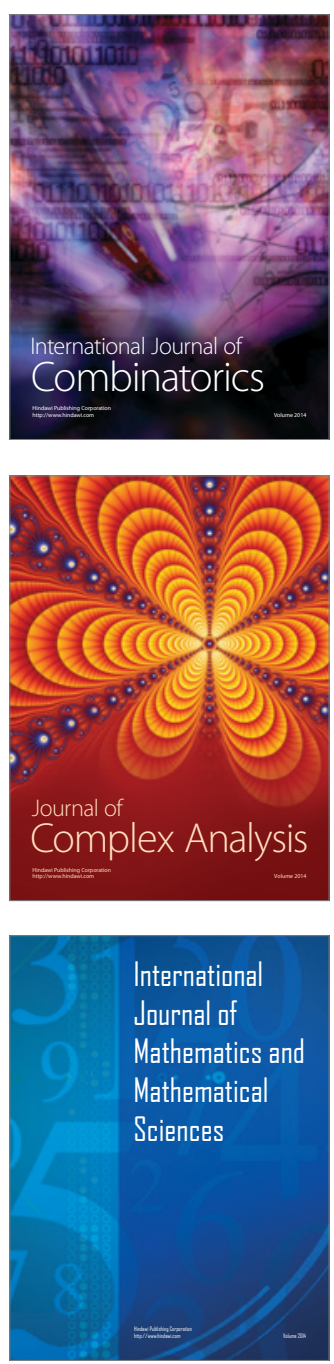
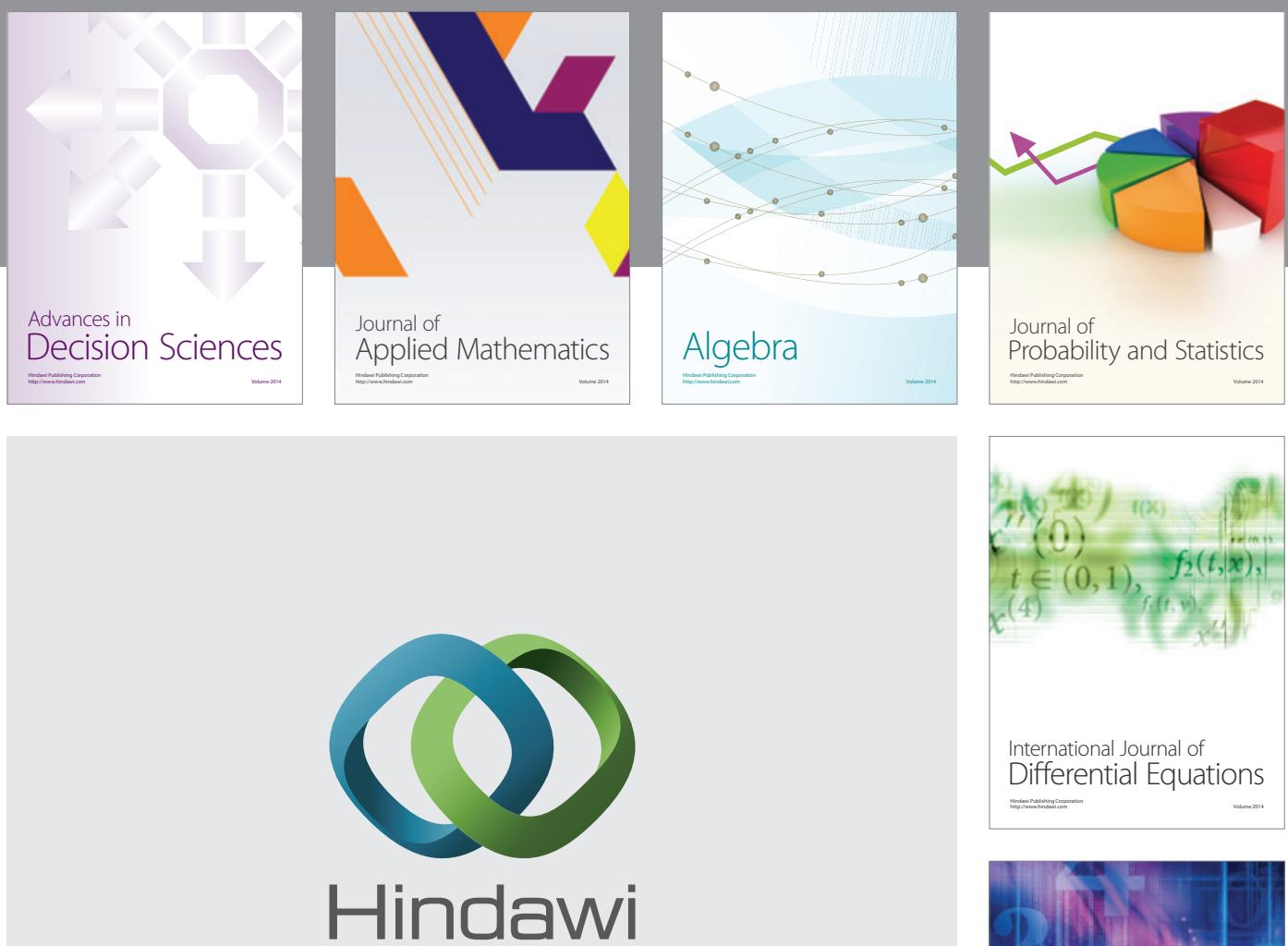

Submit your manuscripts at http://www.hindawi.com
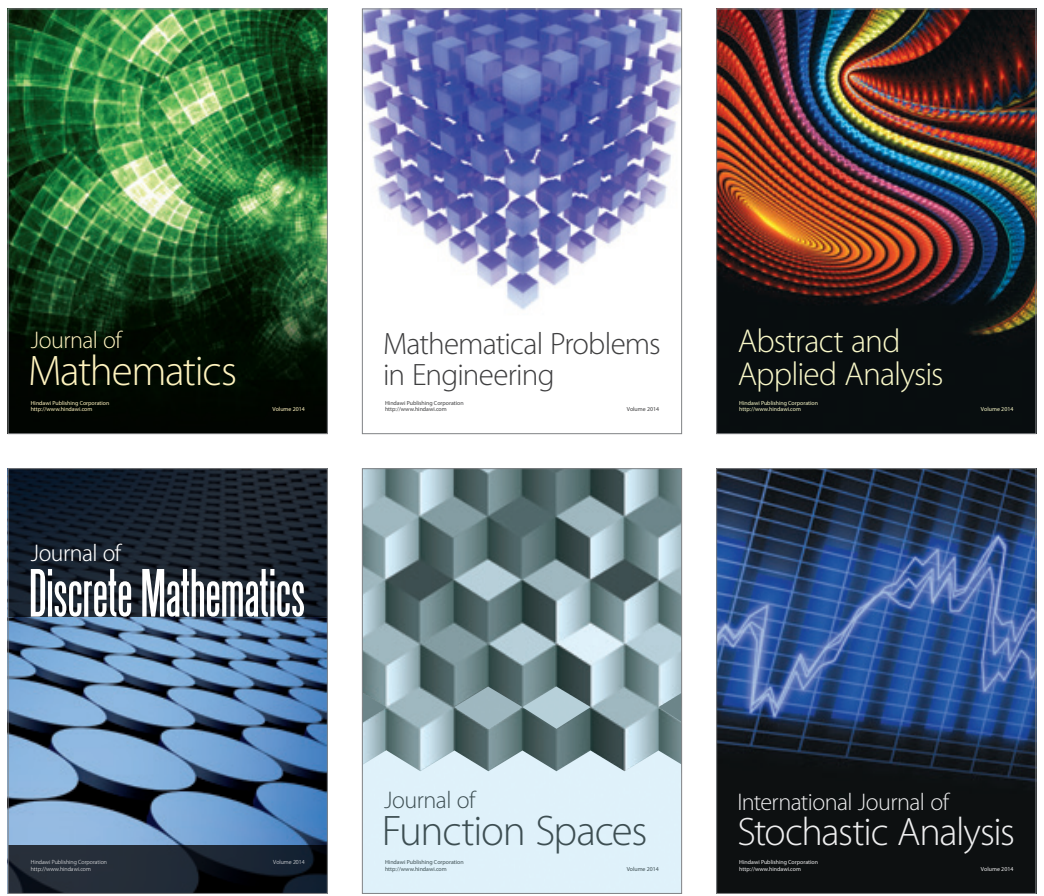

Journal of

Function Spaces

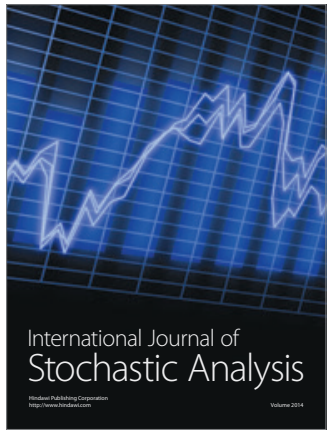

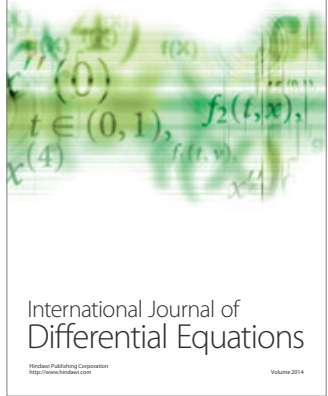
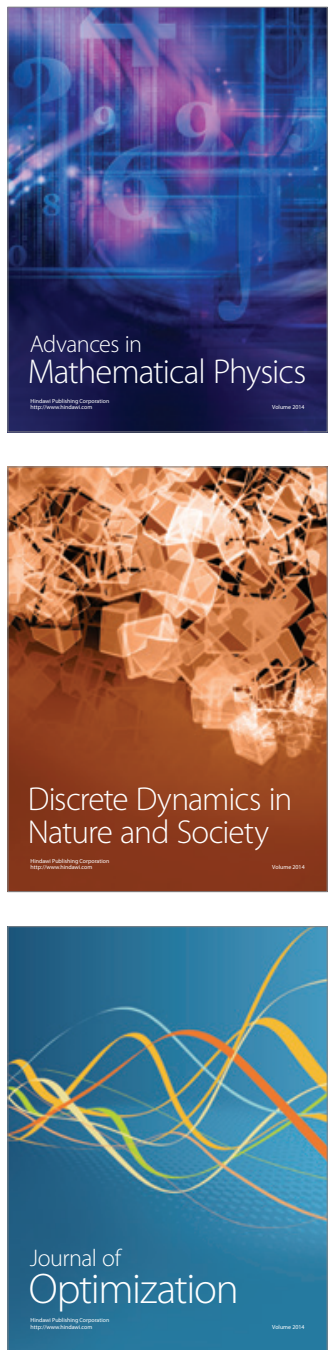